\title{
Two-Stage Residential Energy Management Considering Network Operational Constraints
}

\author{
Benyamin Moradzadeh, Student Member, IEEE, and Kevin Tomsovic, Fellow, IEEE
}

\begin{abstract}
This paper suggests a decentralized optimization of residential demand to minimize the cost to the utility company and customers while maintaining customer satisfaction. As an important feature, distribution system constraints such as equipment capacity, line flow limits, and phase balance constraints are taken into account. Due to the large amount of uncertainty in the residential demand, a two-stage pricing is considered. In the first stage, day-ahead prices as well as residential appliance scheduling are determined through interaction of the utility company and residential customers. Uncertainty of the customer demand is also considered in the day-ahead pricing. In the second stage, on the other hand, prices will be updated based on the actual residential loads. Similar to locational marginal price (LMP) in transmission system, customers on different nodes are charged with different prices. Performance of the approach is shown on a sample distribution system.
\end{abstract}

Index Terms-Decentralized optimization, demand response (DR), dual decomposition, real-time pricing (RTP).

\section{INTRODUCTION}

W HILE DIRECT load control of end-user loads has existed for decades, price driven response programs are only beginning to be explored at the distribution level. These programs utilize a price signal as a means to control the demand [1]. Implementing RTP in transactive markets requires distributed controllers and a centralized auction to create an interactive system which can limit demand at key times on a distribution system and decrease congestion [1].

Due to high utilization of plug-in hybrid electric vehicle (PHEV), there will be a considerable potential for congestion in distribution systems. Congestion can happen in distribution lines and cables or in the form of overload in transformers [2]. The challenge in the DR management is to converge to a solution across the numerous customers while ensuring the utility distribution system satisfies operational and reliability constraints. Much of the literature in this area fails to account for these network constraints.

There exists extensive literature on demand response [3]-[12]. In [3] and [4], residential response management

Manuscript received September 24, 2012; revised March 28, 2013; accepted May 08, 2013. Date of publication July 23, 2013; date of current version November 25, 2013. Paper no. TSG-00625-2012.

The authors are with Center for Ultra-Wide-Area Resilient Electric Energy Transmission Networks (CURENT), EECS Department, University of Tennessee, Knoxville, TN 37996-0001 USA (e-mail: benyamin78@gmail.com; tomsovic@tennessee.edu).

Color versions of one or more of the figures in this paper are available online at http://ieeexplore.ieee.org.

Digital Object Identifier 10.1109/TSG.2013.2265313 is performed only on selected types of appliances. In [5], the authors extend the study to include different appliances in a typical household and propose a method for customers to schedule their available distributed energy resources to maximize net benefits in a day-ahead market. Scenario-based stochastic optimization and robust optimization approaches are developed in [6] to explore optimal real-time DR management decisions with respect to time varying electricity price uncertainties. Authors in [7] proposed a robust linear programming-based approach to schedule the load in presence of real-time price uncertainty. In [8] the electricity price is modeled as a Markov chain with unknown transition probabilities. This model features implicit estimation of the impact of future electricity prices and current control operation on long-term profits. The mentioned papers only optimize the energy consumption of a single smart house to reduce electricity bill for residential customers. However, this may not solve the peak demand issue in residential feeders. For example, if every household desires to shift its load to off-peak times, the energy provider will face a new on-peak period. In other words, it shifts in time rather than limits the peak.

In [9]-[12], researchers include both energy provider and customer benefits. The authors in [9] consider a power network where customers choose their daily schedules of household appliances/loads by playing games among themselves and the utility company tries to adopt adequate pricing tariffs that differentiate the energy usage in time and level to make the Nash equilibrium minimizes the energy costs. However, customer satisfaction is not explicitly represented in the problem formulation. A decentralized optimization based on social welfare maximization is proposed in [10] and [11]. Although customer satisfaction is considered in the optimization, these papers fail to develop appropriate customer utility functions based on different residential appliances. In order to deal with uncertainty of the residential demand, the authors in [13] propose a stochastic sub-gradient algorithm for the day-ahead energy procurement. Nevertheless, due to the large number of residential customers and numerous scenarios associated with random behavior of the customers, stochastic programming is very challenging to implement.

This paper offers a two-stage pricing approach to encourage residential customers to participate in the DR program. The pricing mechanism is similar to the wholesale power market at the transmission level in which there are two markets (dayahead and real-time) for generation units and loads to participate.

In the first stage of the proposed DR management, day-ahead prices are calculated based on social welfare maximization. This 
is a decentralized approach in which home area network management systems (HAN) and distribution utility company exchange information on energy consumption and price. When the optimization converges, the day-ahead prices as well as residential appliance energy consumption patterns are determined. In order to include network operational constraints, nodal pricing is proposed in this paper. This is similar to locational marginal price (LMP) at transmission level. In real-time, the price is calculated based on the actual residential load. Another important consideration of the proposed approach, and frequently ignored in the literature, is to avoid overly sophisticated decision-making at the customer level. Most customers will have limited capacity or need for elaborate scheduling where actual energy cost savings will be modest. Demand uncertainty is another important challenge which will be addressed in the present paper.

\section{SYSTEM MODEL}

Currently, the independent system operators (ISOs)/ regional transmission organizations (RTOs) have visibility into transmission substations, but may have visibility into large sub-transmission substations where large commercial and industrial customer DR programs are located, but generally does not have visibility into the distribution network where most of the small commercial and the main residential DR takes place. Other entities, such as utility distribution companies (UDCs) and loadserving entities (LSEs) interact directly with consumers on the one hand and the ISO/RTO operator on the other hand. They play an important role in bundling the DR from their subscribed customers into products used in the ISO/RTO markets [2]. The utility company purchases electricity from wholesale market and sells it to the customers in the retail market. The system model is described in the following section.

\section{A. Utility Distribution Company}

A small representative section of a residential feeder, including part of the substation is exhibited in Fig. 1[14].

The utility company tends to minimize its cost while ensuring that the distribution system operational constraints are satisfied. The cost function can be expressed as a convex function based on the aggregated residential load supplied by the utility [9]:

$$
C^{t}=\sum_{i=1}^{N_{l}} a\left(P_{i}^{t}\right)^{2}+b P_{i}^{t}+c
$$

where $C^{t}$ is the cost of providing energy at time $t ; P_{i}^{t}$ is the aggregated load on lateral $i$ at time $t ; N_{l}$ is the number of laterals; and $a, b$, and $c$ are constants.

In order to improve system reliability and reducing customer disturbances, the utility company needs to make sure that the network operational constraints such as equipment capacity, line flow limits, and voltage level are not violated. In addition to the mentioned constraints, current and voltage imbalance are the most severe power quality problems in low voltage (LV) distribution networks [15]. An increase in the voltage imbalance can result in overheating and de-rating of all induction motor types of loads and also the distribution transformers [16], [17]. As shown in Fig. 1, residential loads are mostly single-phase.

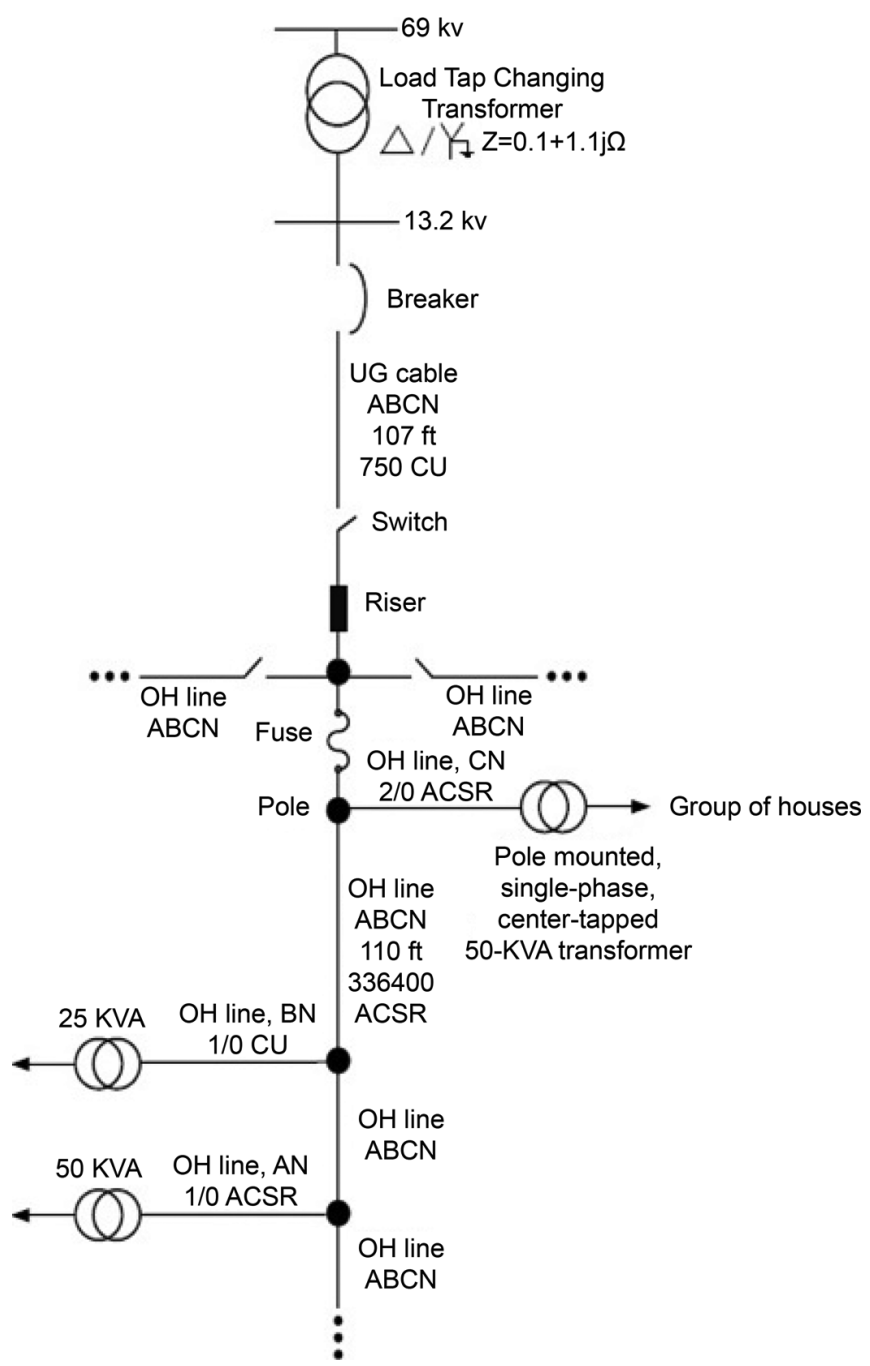

Fig. 1. A small section of a residential feeder.

Therefore, the electric utilities usually try to distribute the residential loads equally among the three phases of distribution feeders [18]. Phase balancing of a 3-phase residential feeder will be very challenging due to the random nature of residential loads. Besides, the magnitude of neutral current of the residential feeders will be very stochastic and may cause random tripping of feeders due to neutral current constraint. Therefore, it is very important for the utility company to mitigate the phase imbalance problem. In this paper, distribution system operational constraints such as equipment capacity, line flow limit, and phase balance constraints are considered in the DR management. The optimization problem (2) minimizes the cost to the utility company subject to the distribution system constraints.

$$
\min \sum_{t=1}^{T} C^{t}
$$

s.t

$$
\begin{aligned}
& \left|\sum_{i=1}^{N_{a}} P_{i, a}^{t}-\sum_{i=1}^{N_{b}} P_{i, b}^{t}\right| \leq \gamma \\
& \left|\sum_{i=1}^{N_{a}} P_{i, a}^{t}-\sum_{i=1}^{N_{c}} P_{i, c}^{t}\right| \leq \gamma
\end{aligned}
$$




$$
\begin{aligned}
& \left|\sum_{i=1}^{N_{b}} P_{i, b}^{t}-\sum_{i=1}^{N_{c}} P_{i, c}^{t}\right| \leq \gamma \\
& A . P_{l}^{t}=P_{i}^{t}, \forall i \\
& 0 \leq P_{i}^{t} \leq P_{i}^{\text {max }} \\
& 0 \leq P_{l}^{t} \leq P_{l}^{\text {max }}
\end{aligned}
$$

where $N_{a}, N_{b}$, and $N_{c}$ are number of $A n, B n$, and $C n$ phase laterals, respectively. $P_{i, a}^{t}, P_{i, b}^{t}$, and $P_{i, c}^{t}$ are residential loads on phases $\mathrm{a}, \mathrm{b}$, and $\mathrm{c}$, respectively. $\gamma$ is the maximum level of acceptable phase imbalance. $A$ is the node-incidence matrix; and $P_{l}^{t}$ and $P_{i}^{t}$ are vectors of line flows and loads on the buses at time $t$, respectively.

Constraints (3)-(5) state that the phase imbalance should stay in a standard range set by the utility company $(\gamma)$. For example, the utility company can set $\gamma$ based on over-capacity of unbalanced transformer (OCUT) index used in [15]. It is worth mentioning that the formulation can easily include 3-phase and 2-phase laterals as well.

Constraint (6) expresses active power balance on every node (for the sake of simplicity the active power loss on the line is not considered). Constraints (7) and (8) formulate the line flow limit and distribution transformer capacity limit, respectively.

\section{B. Residential Loads}

In every household, HAN schedules the appliances to minimize the utility bill while mitigating the impact on the user's comfort. The loads within the residence can be categorized based on whether they can be scheduled to later times. These will be labeled schedulable and non-schedulable loads. For instance, lighting and computer usage are generally non-schedulable loads; while washing machines, heating and air conditioning systems are considered as schedulable loads. Obviously, the agents can only manage schedulable loads. Note some loads can be reduced, such as, lighting, for periods of time at the cost of convenience and would normally be done at times of extreme shortage. For simplicity, these are not modeled here. In this paper, residential loads are divided into five classes. In the following section, the detailed model of a smart home is presented.

1) Class 1: This class includes non-schedulable appliances such as refrigerator-freezer, electric stove, lighting, TV, computer, etc. As mentioned before, one of the important features of the pricing algorithm is to avoid burdening the customers. Since it will be very inconvenient for the customers to forecast their usage of the appliances in this class, it is assumed that the HANs will estimates the energy consumption of this class based on statistic data and parameters such as number of people in the house, day of the week, and season. This information can be estimated based on the customer behavior during the last few days. Note that the information can also be entered by the customers if they want to. Because of the inaccurate prediction of the energy usage of class 1 appliances, a normal distribution function is used to model uncertainty of the forecast. Normal distribution function has been widely used in load forecasting in power systems [19]. Suppose that $E_{1}^{t}$ is the estimated energy consumption of class 1 appliances in a house. Then, the actual energy consumption of non-schedulable loads is obtained by:

$$
\widetilde{E_{1}^{t}}=E_{1}^{t}+\delta, \delta=\aleph\left(0, \sigma_{1}^{2}\right)
$$

where $\widetilde{E_{1}^{t}}$ is the actual load and $\delta$ is the forecast error with zero mean and variance $\sigma^{2}$.

2) Class 2: This class contains devices which have a prescribed energy requirement, $E$, that has to be completed over $T$ time slots, starting from time $t_{1}$. An example is charging of a PHEV, where the user may specify the time for charging to start and required completion time. For example, a PHEV sedan for a 40-mile daily driving range needs $E=16 \mathrm{kWh}$ in the battery [20]. The PHEV utility function considered in this paper is as follows:

$$
U(q)=\alpha_{E V}\left(E-q^{e n d}\right)^{2}
$$

where $q^{\text {end }}$ is the total energy given to the battery by the end of charging period, and $\alpha_{E V}$ is the weighting factor determined based on customer preference. The charge level at each time slot is given by:

$$
\begin{aligned}
& q(\tau)=q^{i n i t}+\sum_{t=t_{1}}^{\tau} p_{b}(t) \cdot t \\
& p_{b}^{\text {min }}<p_{b}^{t}<p_{b}^{\text {max }}
\end{aligned}
$$

where $p_{b}^{t}$ is the charging rate at time $t . p_{b}^{\text {min }}$ and $p_{b}^{\text {min }}$ are minimum and maximum charging rates, respectively.

3) Class 3: This class includes thermal loads such as heating, ventilation and air-conditioning (HVAC), water heater, and cooled water reservoir, with temperature profile $\theta$, which must be kept within minimum and maximum temperature limits, $\theta_{\min }$ and $\theta_{\max }$. The temperature of the heat store evolves according to:

$$
\theta(t)=\theta(t-1)+\alpha\left(\theta^{a m b}(t)-\theta(t-1)\right)+\beta E^{t}
$$

where $\theta^{a m b}(t)$ is the ambient temperature profile; $E^{t}$ is the energy consumption of the thermal load at time $t$; and $\alpha$ and $\beta$ are parameters that specify the thermal characteristics of the appliance and the environment where it operates. This formulation models the fact that the current temperature depends on the current power draw as well as the temperature in the previous time-slot [21], [22].

The utility function is developed based on the deviation of the temperature from customer setting as follows:

$$
\begin{aligned}
& \alpha_{a c}\left(\theta(t)-\theta^{s e t}\right)^{2} \\
& \theta_{\min } \leq \theta(t) \leq \theta_{\max }
\end{aligned}
$$

where $\alpha_{a c}$ is the determined based on how customer cares about the temperature.

4) Class 4: This class of appliances includes deferrable loads. These loads must consume a minimum amount of power over a given interval of time, which is characterized by the 
constraint $\sum_{t \in \tau} p_{l}(t) \geq E$, where $E$ is the minimum total consumption for a certain period of time. In some cases, such as dishwasher and cloth washer/dryer, the load can only be turned on or off in each time period. These appliances consume more or less fixed power while they are on. The primary interest of customers is that the work is done by a certain time. Therefore, based on the electricity price and the resident's comfort, the HAN decides when to turn the device on.

5) Class 5: This class of appliances includes battery storage units which can be used to maximize the utilization of the residential renewable energy resources such as rooftop solar panels. The utility function of a battery is considered as follows:

$$
U(q)=\alpha_{1}\left(P_{b}^{t}\right)^{2}-\alpha_{2} P_{b}^{t} P_{b}^{t+1}
$$

where $\alpha_{1}$ and $\alpha_{2}$ are positive constants and $P_{b}^{t}$ is the battery output power at time $t$. The first term captures the damaging effect of fast charging and discharging; and the second term penalizes charging/discharging cycles. The charge/discharge constraints are formulated as follows:

$$
\begin{aligned}
& -\left(1-S C_{b}^{t-1}\right) C_{b} \leq P_{b}^{t} t \leq\left(S C_{b}^{t-1}-D_{b}\right) C_{b} \\
& -P_{c h, \max } \leq P_{b}^{t} \leq P_{d c, \max }^{b}
\end{aligned}
$$

where $S C_{b}$ is the state of the charge of the battery, $D_{b}$ is the minimum acceptable energy in the battery, and $C_{b}$ is the capacity of the battery. In order to preserve battery's life, (6) guarantees shallow discharge.

\section{PRICING MECHANISM}

We are looking for a decentralized DR in which HANs at the residences communicate with the utility company through Advanced Metering Infrastructures (AMI). HANs receive the prices for the next 24 hours from the utility company and schedule residential appliances based on the hourly prices and customers' utility functions. Then, the day-ahead prices are updated based on the messages sent to the utility distribution company by the HANS. As mentioned earlier, even though uncertainty of the demand is considered in the day-ahead optimization, due to the highly unpredictable behavior of the customers day-ahead prices do not exactly reflect the actual residential loads. Therefore, the prices need to be updated in real-time. This paper offers a two-stage pricing approach to encourage residential customers to participate in the DR management program. In the first stage, day-ahead prices are calculated based on social welfare maximization. The second stage determines the real-time prices based on the actual residential loads.

\section{A. Day-Ahead Pricing}

From a social fairness point of view, it is desirable to minimize the cost to the energy provider while mitigating the impact on the user's comfort. The social welfare maximization is mathematically formulated as follows:

$$
\min \sum_{t=1}^{T}\left(\sum_{i=1}^{N_{i}} C\left(P_{i}^{t}\right)+\sum_{i=1}^{N_{i}} \sum_{j=1}^{N_{j}} \sum_{k=1}^{N_{k}} D_{k, j, i}^{t}\right)
$$

$$
\text { s.t } \begin{aligned}
& \sum_{j=1}^{N_{j}} \sum_{k=1}^{N_{k}} E_{k, j, i}^{t} \leq P_{i}^{t}, \forall i \\
& (3)-(8) \\
& \text { class }(2)-(5) \text { constraints }
\end{aligned}
$$

where $P_{i}^{t}$ is the aggregated load on node $i ; D_{k, j, i}^{t}$ and $E_{k, j, i}^{t}$ are disutility (discomfort) function and energy consumption corresponding to the $k^{t h}$ appliance in $j^{t h}$ house which is located on node $i$. Equation (18) guaranties the supply-demand balance on every node. Equation(19) includes the network operational constraints (3)-(8). Equation (20) includes constraints corresponding to different classes of residential loads explained in Section II-B. where $P_{i}^{t}$ is the aggregated load on node $i$; $D_{k, j, i}^{t}$ and $E_{k, j, i}^{t}$ are disutility (discomfort) function and energy consumption corresponding to the $k^{t h}$ appliance in $j^{t h}$ house which is located on node $i$. Equation (18) guaranties the supply-demand balance on every node. Constraint (19) includes the network operational constraints (3)-(8). Constraint (20) includes constraints corresponding to different classes of residential loads explained in Section II-B.

When load uncertainty exists due to the stochastic behavior of the residential customers, the optimization problem (17) becomes minimization of the expected value of the objective function (stochastic optimization model). In order to avoid sophisticated algorithms at the residence, we only consider the uncertainty associated with class 1 appliances in the day-ahead market. The uncertainty of the other load types will be addressed in the real-time price adjustment program. Since no utility function is considered for class 1 appliances, the uncertainty only affects constraint (18). Different uncertainty models can be considered. In this paper, a normal distribution is used to model uncertainty of the demand forecast for class 1 appliances. The energy provider guarantees to supply the residential demand. In other words, the probability of power shortage should be small. Mathematically,

$$
\operatorname{Pr}\left(\sum_{k, j} \widetilde{E_{k, j, i}^{t}}-P_{i}^{t} \geq \eta\right) \leq \varepsilon, \forall i
$$

where $\eta$ is a specified threshold indicating the amount of supply shortage and $\varepsilon$ is a small positive value [23]. It is assumed that $\delta$ is an independent variable for different houses. Therefore, the aggregated $\delta$ over $N_{j}$ houses will be calculated as follows:

$$
\delta_{a g g}=\sum_{j=1}^{N_{j}} \delta_{j} \sim \aleph\left(0, \sum_{j} \sigma_{j}^{2}\right) .
$$

Therefore, (21) turns into:

$$
\begin{aligned}
& \operatorname{Pr}\left(\sum_{k, j} E_{k, j, i}^{t}+\delta_{a g g}-P_{i}^{t} \geq \eta\right) \leq \varepsilon, \forall i \\
& \sum_{k, j} E_{k, j, i}^{t} \leq P_{i}^{t}+\eta-\left(\sqrt{\sum_{j} \sigma_{j}^{2}}\right) Q^{-1}(\varepsilon)
\end{aligned}
$$


where $Q(x)=1 / \sqrt{2 \pi} \int_{x}^{\infty} \exp \left(-u^{2} / 2\right) d u$. In addition to the forecasted demand, every HAN needs to report its $\delta$ to the utility company. Despite the fact that the centralized optimization problem (17) seems easy to solve, the communication overhead requirements create concerns. Moreover, customers generally want to preserve their privacy and may wish to withhold detailed information on energy consumption to the utility company. Therefore, a decentralized optimization method seems both preferable and more practical to implement. This can be done through deployment of advanced metering infrastructures (AMI) and HANs.

A global solution can be efficiently found for a convex optimization problem. However, when the network is not convex, even finding a feasible solution becomes difficult. The optimization problem (17) is convex, except for class 4 residential loads which are on/off devices. We simply relax the integer constraints (on/off) and convert the integer programming optimization problem into linear programming. An approximate solution can be obtained by rounding the solutions of the relaxed problem into the nearest integers.

The convex problem (17) has a separable structure. Therefore, it can be solved in a decentralized way through dual decomposition and sub-gradient method. Keeping the rest of the constraints implicit, the Lagrangian function for (17) is given by:

$$
\begin{aligned}
L\left(\left\{\lambda_{i}^{t}\right\},\left\{E_{k, j, i}^{t}\right\},\right. & \left.\left\{P_{i}\right\}\right)=\sum_{t=1}^{T} \sum_{i=1}^{N_{l}} C\left(P_{i}^{t}\right)+\sum_{t=1}^{T} \sum_{i, j, k} D_{k, j, i}^{t} \\
& +\sum_{t=1}^{T} \sum_{i=1}^{N_{l}}\left(\lambda_{i}^{t} B_{i}^{t}\right)
\end{aligned}
$$

$\min L\left(\left\{\lambda_{i}^{t}\right\},\left\{E_{k, j, i}^{t}\right\},\left\{P_{i}\right\}\right)$

where $B_{i}^{t}=\sum_{j, k} E_{j, k, i}^{t}(l)-P_{i}^{t}(l)-\eta+\left(\sqrt{\sum_{j} \sigma_{j}^{2}}\right) Q^{-1}(\varepsilon)$. $\lambda_{i}^{t}$ denotes the Lagrange multiplier corresponding to constraint (18). The Lagrangian minimization (26), is easily seen to be decomposed to optimizations at the utility level and residents. Specifically, the dual decomposition method consists of the following iterations, indexed by $\ell=1,2, \ldots$ and initialized with arbitrary $\lambda_{i}^{t} \geq 0$, and $\beta_{i}$ as the step size.

$$
\min \left(\sum_{i=1}^{N_{l}} C\left(P_{i}^{t}\right)-\sum_{i=1}^{N_{l}} \lambda_{i}^{t}(l) P_{i}^{t}(l)\right), \forall i
$$

s.t

$$
\min \left(D_{k, j, i}^{t}(l)+\lambda_{i}^{t} E_{j, k, i}^{t}(l)\right), \forall i, j, k
$$

s.t

$$
\text { class }(2)-\operatorname{class}(5) \text { constraints. }
$$

The Lagrangian multiplier for each node is updated according to:

$$
\lambda_{i}^{t}(l+1)=\max \left[\lambda_{i}^{t}(l)+\beta_{i} B_{i}^{t}, 0\right], \forall i .
$$

The Lagrangian multipliers are considered as the nodal prices. This is a similar concept to LMP in transmission system. Through setting the nodal prices, the utility company can involve the customers in mitigating violations in network operational constraints. For example, when a transformer overload happens, the customers on the corresponding lateral will be penalized with extra charge due to their exceeding energy usage. The higher price encourages the customers to reduce their load [according to (29)]. This concept will be demonstrated and discussed in the simulation results.

The optimization problem (27) is performed by the utility agent over $t=1$ : $T$. Equation (27) maximizes the utility company's profit with respect to the distribution grid constraints. Optimization (29), which is performed by HANs at the residence, minimizes the customers electricity bill as well as disutility function. The Lagrangian multipliers, corresponding to nodal prices, are updated in each iteration according to (31). This process repeats until $B_{i}^{t}$ is smaller than a specified threshold for every node $i$ (a small positive number).

Convergence of iterative approach (27) can be obtained for the following three step size rules: constant step size, non-summable but square-summable step size, and step size given by harmonic series. Convergence of the subgradient method with these step size rules is studied in the literature. The related results are summarized and discussed in [11].

In order to guarantee convergence, primal averaging is necessary if the objective function is neither strictly convex nor finite. It should also be used when the objective is not a function of all optimization variables [11]. In this problem, the primal objective function is strictly convex and finite. However, it is not a function of all optimization variables, which is a consequence of considering different device classes (utility functions are not defined for some appliance classes). Therefore, running average method is applied for all the variables, e.g., $E_{j, k}^{\bar{t}}=1 / l \sum E_{j, k}^{t}$. Authors in [11] prove that the algorithm finds near-optimal schedules even when AMI messages (updated prices and residential load) are lost, which can happen in the presence of malfunctions or noise in the communications network. It is worth noting that when the primal objective function is not strictly convex and/or finite, the alternating direction method of multipliers (ADMM) can be used to guarantee convergence [24].

\section{B. Real-Time Price Adjustment}

As mentioned before, because of the highly random behavior of the residential customers a price adjustment in real-time is needed to reflect the actual residential load. Customers participate in the day-ahead market and purchase electricity as discussed in the last section. In the real-time, the price is calculated based on the actual residential load. This is similar to optimal power flow (OPF) in transmission system.

For a resident who participates in day-ahead market, in case that real-time price is higher than the day-ahead price, he will pay the same price as the day-ahead price. However, if the residence consumes more energy as he promised in the day-ahead, he has to pay for the extra energy with the real time price. In other words, the customers participating in the day-ahead market are charged with the minimum price between day-ahead and real-time price.

In order to encourage customers to accurately/honestly report their estimated energy consumption in day-ahead, a penalty can 


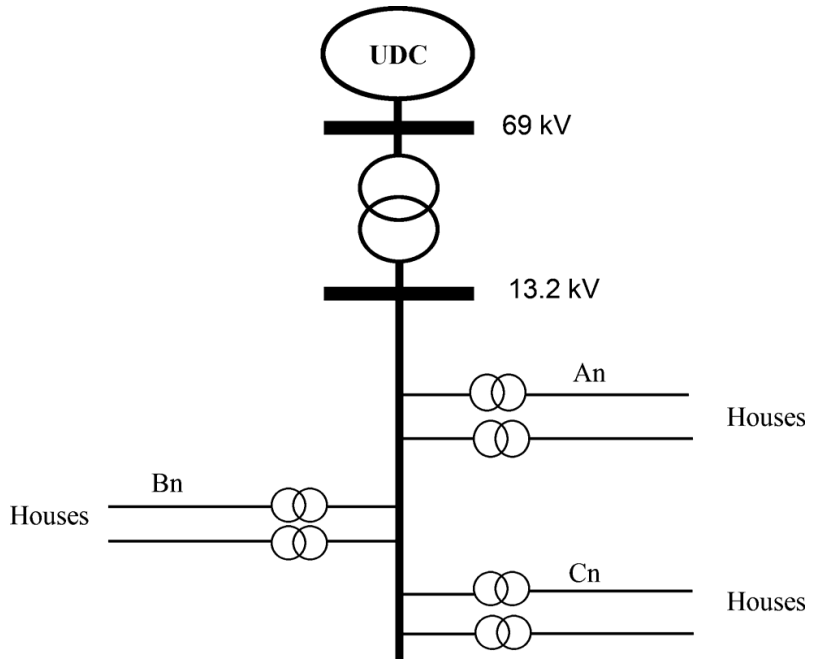

Fig. 2. Topology of the test system.

be set by the utility company to penalize large difference between day-ahead and real-time energy consumption of the residences which can be expressed by:

$$
\left\|E_{d h}-E_{r t}\right\| \leq \varphi
$$

where $E_{d h}$ is the hourly energy consumption scheduled by a residential customer in day-ahead and $E_{r t}$ is the hourly demand of the residence in real-time. $\varphi$ is a threshold set by the utility company.

Through encouraging the customers to participate in the dayahead market, utility company can have a better estimation on the residential load and plan to cope with potential violation in distribution system operational constraints such as transformer overload and congestion.

\section{Simulation Results}

The simulations are performed on a $2.66 \mathrm{GHz}, 4 \mathrm{~GB}$ RAM PC. The software tools used for the simulations are MATLAB 2011b and ILOG CPLEX 12.2 under Windows 7 operation system. Performance of the proposed algorithm is demonstrated on a simple system with 30 residential customers.

Configuration of the test system is exhibited in Fig. 2 with 6 residential laterals and equal number of houses on each lateral. It is assumed that the system two $A n$, two $B n$, and two $C n$ phase laterals. In our simulation, $b^{t}=c^{t}=0$, and $a^{t}=0.5$ are considered for the utility company cost function in (1).

Different types of customers are considered in the simulations. For example, some customers are out of their houses during the working hours while some other stay in their house the entire day. Each household is also assumed to have schedulable appliances such as PHEV, air conditioner, washer/dryer, and dishwasher. Different houses use different weighting factors for utility functions corresponding to the residential appliances.

The optimization is performed over 24 hours time horizon. Fig. 3 shows the convergence of the approach on the feeder at

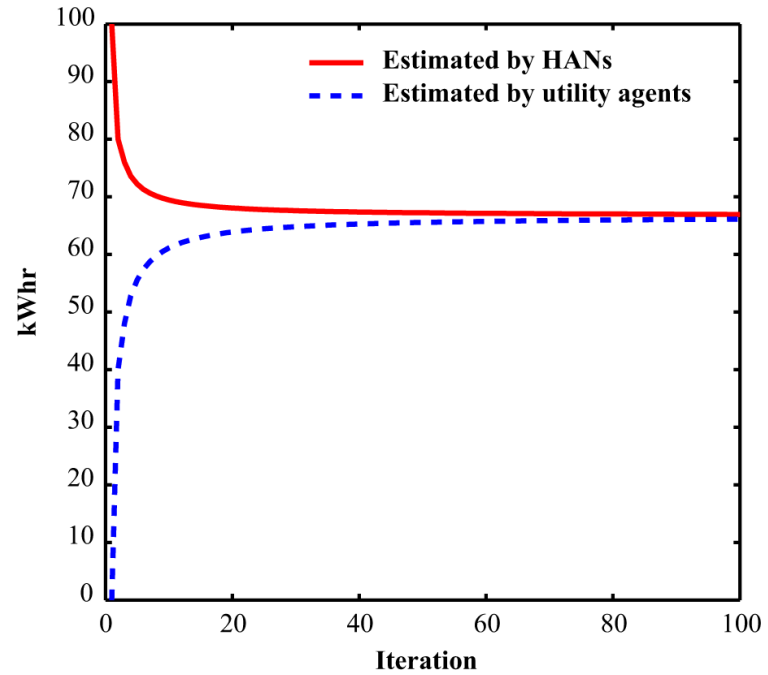

Fig. 3. Convergence of the algorithm at the residential feeder.

6:00 P.M. Hourly nodal prices and residential appliance scheduling over 24 hours will be obtained at the point of convergence. It is worth noting that different time intervals, e.g., 15 minutes, can also be considered.

A small constant stepsize $(\beta=0.15)$ is chosen for the subgradient updates, coupled with dual and primal averaging, in order to obtain near-optimal dual and primal values in a finite number of iterations. As observed in Fig. 3, the decentralized optimization is converged after several iterations. Note that the utility company and HANs exchange small size of information (price and residential energy consumption). The power schedule updates occur in parallel across all residential customers and therefore the computation time per iteration is the maximum time, over the utility company and all houses, to solve their optimization problem. Therefore, speed of the process does not depend on the number of the customers. Moreover, since simple models of residential appliances are considered, the optimization performed by HANs is fast. In our test case, it takes 0.2 seconds for the algorithm to converge.

Fig. 4-Fig. 7 show the effect of distribution system operational constraints on nodal prices Fig. 4-Fig. 5 show the change in nodal price $(\notin / k W h)$ on bus 6 due to distribution transformer capacity constraint. As shown in Fig. 4, the aggregated load on node 6 is reduced to avoid transformer overload. When distribution transformer overload happens, the decentralized optimization approach automatically increases the nodal price on bus 6 (Fig. 5). Therefore, some customers prefer to reduce their energy consumptions. For example, if a customer has not assigned a high weighting factor for HVAC, the energy consumption of the HVAC will be reduced by HAN (the temperature deviates from the set point but still remains in the comfortable range). This is because the HAN minimizes the customer's utility bill as well as disutility function according to (29). Similar situation will happen in case of line congestion. For example, when a distribution line is congested, customers on the downstream nodes will be penalized with extra charge due to their exceeding energy consumption. The higher prices 


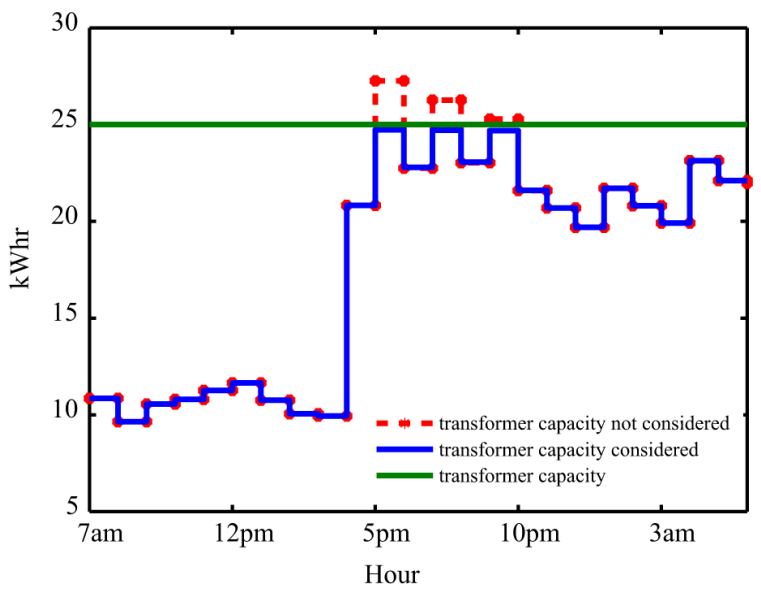

Fig. 4. Effect of transformer capacity constraint on residential loads.

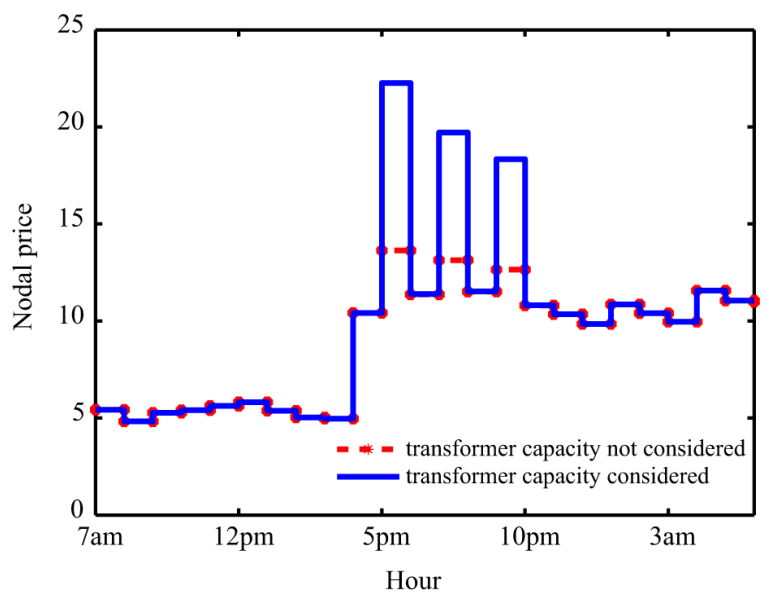

Fig. 5. Effect of transformer capacity constraint on nodal prices.

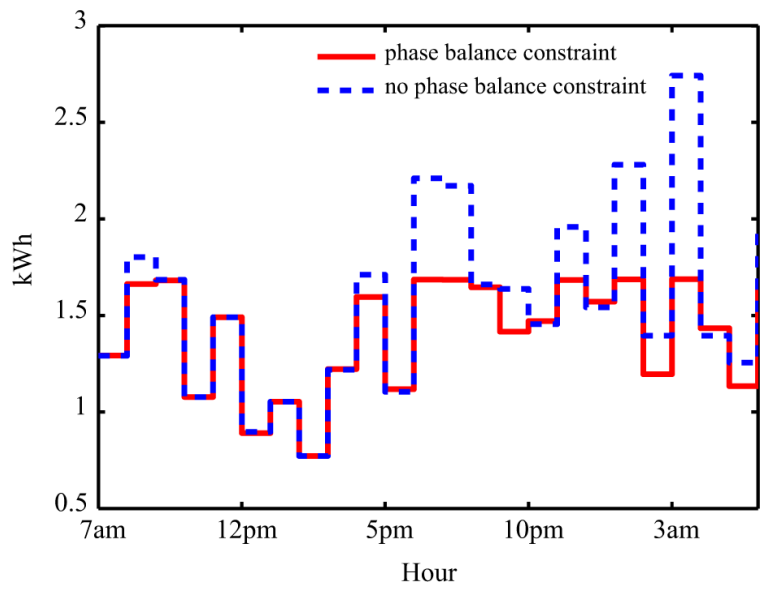

Fig. 6. Effect of phase balance constraint on residential loads.

change the solution of (29) in such a way that the network operational constraint violation is prevented.

Fig. 6-Fig. 7 show the effect of phase balance constraints on the optimization results, i.e., residential load and nodal prices. Fig. 6 exhibits the difference of loads on phases $a$ and $c$ $\left(\left|\sum_{i=1}^{N_{a}} P_{i, a}^{t}-\sum_{i=1}^{N_{c}} P_{i, c}^{t}\right|\right)$ when the phase balance constraints is included. As shown in Fig. 7, the nodal prices $(\phi / k W h)$ on the phases change in such a way that (3)-(5) are satisfied. For example, if phase $c$ has a higher load than phase $a$ in such

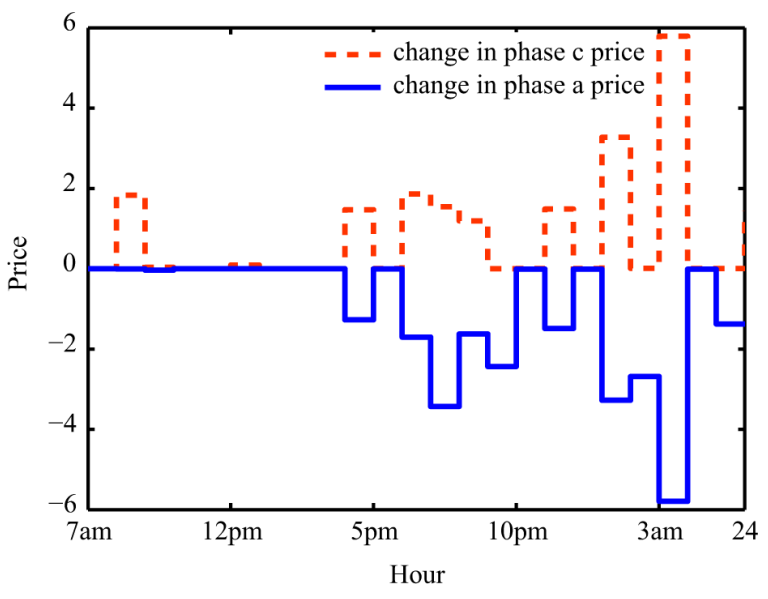

Fig. 7. Effect of phase balance constraint on nodal prices.

a way that $\left|\sum_{i=1}^{N_{a}} P_{i, a}^{t}-\sum_{i=1}^{N_{c}} P_{i, c}^{t}\right|>\gamma,(\gamma=1.7 \mathrm{~kW}$ in the simulations), the nodal prices on the laterals connected to phase $c$ will increase while the nodal prices on the nodes supplied by phase $a$ decrease. As the price changes, the result of optimization problem (29) for the residences located on the mentioned phases will change. Similar results will be obtained for phases $a-b$, and $b-c$.

It is worth noting that the distribution system operational constraints can also be included as soft constraints. In other words, the objective function of the utility company can include these soft constraints by assigning different weighting factors. Therefore, the operational constraints such as phase balance can be violated but the customers will have to pay more money depending on the weighting factors set by the utility company. In the second stage, the day-ahead prices are updated according to the discussion in Section III-B. The customers who participate in the day-ahead market have the advantage to pay less for their promised energy consumption. Moreover, to encourage the customers to honestly/accurately report their day-ahead demand, a penalty function based on the difference between hourly day-ahead and real-time demands can be set by the utility company.

\section{CONCLUSIONS}

In this paper, a two-stage pricing mechanism is proposed for residential demand response management. In the first stage, day-ahead prices and residential load schedule are obtained through a decentralized optimization. Day-ahead pricing is very important as it helps the utility company to have a good estimation on the load for the next day. The decentralized optimization is developed using dual decomposition and sub-gradient method.

The objective of the day-ahead optimization is minimizing the cost to the utility company and residential customers while maintaining customer satisfaction. Five different classes of the residential appliances are considered and detailed models are developed accordingly. Since the energy consumption of nonschedulable residential loads is challenging to forecast, a normal distribution function is used to model the uncertainty of the estimation. As an important contribution of the paper, distribution system operational constraints such as equipment capacity, 
line flow limits, and phase balance are taken into account in the pricing mechanism. Effect of the operational constraints on the nodal prices are demonstrated and discussed.

Due to the highly random energy consumption at the residence, the second stage of pricing is used to adjust the price in real-time. The real-time price reflects the actual residential load. An extension to this work can be achieved through including distributed renewable resources owned by the utility company and/or residents. The demand response management program should help to maximize the utilization of these stochastic energy resources.

\section{REFERENCES}

[1] N. W. Miller, K. Clark, and M. Shao, "Frequency responsive wind plant controls: impacts on grid performance," in Proc. 2011 IEEE Power Energy Soc. Gen. Meet., pp. 1-8.

[2] F. Rahimi and A. Ipakchi, "Demand response as a market resource under the smart grid paradigm," IEEE Trans. Smart Grid, vol. 1, pp. 82-88, Jun. 2010.

[3] P. Du and N. Lu, "Appliance commitment for household load scheduling," IEEE Trans. Smart Grid, vol. 2, pp. 411-419, Jun. 2011.

[4] K. Clement-Nyns, E. Haesen, and J. Driesen, "The impact of charging plug-in hybrid electric vehicles on a residential distribution grid," IEEE Trans. Power Syst., vol. 25, pp. 371-380, Feb. 2010.

[5] M. Pedrasa, T. Spooner, and I. MacGill, "Coordinated scheduling of residential distributed energy resources to optimize smart home energy services," IEEE Trans. Smart Grid, vol. 1, pp. 134-143, Sep. 2010.

[6] Z. Chen and L. Wu, "Real-time price-based demand response management for residential appliances via stochastic optimization and robust optimization," IEEE Trans. Smart Grid, vol. 3, pp. 1822-1831, Dec. 2012.

[7] A. J. Conejo, M. J. Morales, and L. Baringo, "Real-time demand response model," IEEE Trans. Smart Grid, vol. 1, pp. 236-242, Dec. 2010.

[8] T. T. Kim and H. Vincent Poor, "Scheduling power consumption with price uncertainty," IEEE Trans. Smart Grid, vol. 2, pp. 519-527, Sep. 2012.

[9] A. H. Mohsenian-Rad, V. S. W. Wong, J. Jatskevich, R. Schober, and A. Leon-Garcia, "Autonomous demand side management based on game theoretic energy consumption scheduling for the future smart grid," IEEE Trans. Smart Grid, vol. 1, pp. 320-331, Dec. 2010.

[10] P. Samadi, A.-H. Mohsenian-Rad, R. Schober, V. W. S. Wong, and J. Jatskevich, "Optimal real-time pricing algorithm based on utility maximization for smart grid," in Proc. 2010 IEEE Int. Conf. Smart Grid Commun., pp. 415-420.

[11] N. Gatsis and G. B. Giannakis, "Residential load control: Distributed scheduling and convergence with lost AMI messages," IEEE Trans. Smart Grid, vol. 3, no. 2, pp. 770-786, Jun. 2012.

[12] M. Fahrioglu and F. L. Alvarado, "Using utility information to calibrate customer demand management behavior models," IEEE Trans. Power Syst., vol. 16, pp. 317-322, Jun. 2002.
[13] L. Jiang and S. Low, "Multi-period optimal procurement and demand responses in the presence of uncertain supply," in Proc. 2011 Conf. Decision Control and Eur. Control Conf. (CDC-ECC), pp. 4348-4353.

[14] P. Jahangiri, D. Wu, W. Li, D. C. Aliprantis, and L. Tesfatsion, "Development of an agent-based distribution test feeder with smart-grid functionality," in Proc. IEEE Power Energy Soc. Gen. Meet., pp. 1-8.

[15] M. T. Bina and A. Kashefi, "Three-phase unbalance of distribution systems: Complementary analysis and experimental case study," Int. J. Elect. Power Energy Syst., vol. 33, pp. 817-826, May 2010.

[16] P. Gnacinski, "Windings temperature and loss of life of an induction machine under voltage unbalance combined with over- or undervoltages," IEEE Trans. Energy Convers., vol. 23, pp. 363-371, 2008.

[17] P. Pillary and M. Manyage, "Loss of life in induction machines operating with unbalanced supplies," IEEE Trans. Energy Convers., vol. 21, pp. 813-822, Dec. 2006.

[18] A. V. Jouanne and B. Banerjee, "Assessment of voltage imbalance," IEEE Trans. Power Del., vol. 16, pp. 782-790, Oct. 2001.

[19] R. Bo and F. Li, "Probabilistic LMP forecasting considering load uncertainty," IEEE Trans. Power Syst., vol. 24, pp. 1279-1289, Aug. 2009.

[20] A. Ipakchi and F. Albuyeh, "Grid of the future," IEEE Power Energy Mag., vol. 7, pp. 52-62, Apr. 2009.

[21] P. Xu, P. Haves, M. A. Piette, and L. Zagreus, "Demand shifting with thermal mass in large commercial buildings: Field tests, simulation and audits," California Energy Commission, Tech. Rep., Jan. 2006 [Online]. Available: http://green.sba.gov/demand-shifting-thermalmass-large-commercial-buildingsfield-tests-simulation-and-audits

[22] J. E. Braun, "Load control using building thermal mass," J. Solar Energy Eng., vol. 125, pp. 292-392, Aug. 2003.

[23] P. Tarasak, "Optimal real-time pricing under load uncertainty based on utility maximization for smart grid," in Proc. 2011 Int. Conf. Smart Grid Commun., pp. 321-326.

[24] M. Kraning, E. Chu, J. Lavaei, and S. Boyd, "Message passing for dynamic network energy management," [Online]. Available: http://www. stanford.edu/boyd/papers/pdf/decen_dyn_opt.pdf

Benyamin Moradzadeh (S'09) received his B.S. from K. N. Toosi University of Technology, Tehran, Iran, in 2004, and his M.S. from Amirkabir Universrity of Technology (Tehran Polytechnic) in 2007. He is currently a Ph.D. student in electrical engineering at the University of Tennessee, Knoxville, TN, USA. His main interests include smart grids and optimization methodologies applied to power system problems.

Kevin Tomsovic (F'07) received the B.S. degree in electrical engineering from Michigan Technological University, Houghton, MI, USA, in 1982 and the M.S. and Ph.D. degrees in Electrical Engineering from the University of Washington, Seattle, WA, USA, in 1984 and 1987, respectively. Currently, he is Head and CTI Professor of the Department of Electrical Engineering and Computer Science at University of Tennessee, Knoxville, TN, USA, where he also directs the NSF ERC CURENT. He was on the faculty of Washington State University from 1992 to 2008. He held the Advanced Technology for Electrical Energy Chair at Kumamoto University, Kumamoto, Japan, from 1999 to 2000 and was an NSF program director in the ECS division of the Engineering directorate from 2004 to 2006. 